\title{
Vom Elysée-Vertrag zum Vertrag von Aachen: Lektionen der Vergangenheit für die Herausforderungen der
}

Gegenwart

Olaf Leiße

\section{Einleitung}

Das Jahr 2019 markiert eine Zäsur in den deutsch-französischen Beziehungen, denn seitdem leben beide Staaten seit dem Ende des Zweiten Weltkriegs genauso lange in Frieden miteinander, wie sie sich zuvor bekämpft haben. Mit dem deutsch-französischen Krieg von 1871/72 vertiefte sich die Erbfeindschaft zwischen beiden Ländern und es begann eine lange Phase praktisch ununterbrochener diplomatischer und kriegerischer Auseinandersetzungen, die nicht nur Politik und Wirtschaft betrafen, sondern auch die Menschen und Völker entfremdeten. Nach dem Zweiten Weltkrieg fand die nunmehr gewünschte Versöhnung ihren ersten Höhepunkt im Schuman-Plan von 1950 und der darauf folgenden Gründung der Europäischen Gemeinschaft für Kohle und Stahl, gefolgt von der Gründung der Europäischen Gemeinschaften 1958. Mit dem Elysée-Vertrag von 1963 setzte sich die Wiederannäherung zwischen Deutschland und Frankreich fort. Dieser Vertrag war zwar nicht der Startschuss, aber der Grundstein für die zukünftige Gestaltung der deutsch-französischen Beziehungen in den folgenden Jahrzehnten. Höhepunkte der Beziehungen zwischen beiden Ländern waren die Feierlichkeiten zum 40-jährigen Jubiläum im Jahr 2003 und zum 55-jährigen Jubiläum im Jahr 2018. Beide Ereignisse sollen im Folgenden vergleichend in den Blick genommen werden. Dabei zeigen sich interessante Unterschiede hinsichtlich der Vertragsausgestaltung und im historischen Umfeld.

\section{Gründung und Grundideen}

Die Präambel des Vertrags zur Gründung der Europäischen Wirtschaftsgemeinschaft formuliert prägnant, dass die Gemeinschaft als »Europa der Staaten und Bürger" gegründet werden soll. Damit nimmt die europäische Integration eine Sonderstellung ein. Während internationale Organisatio- 
nen meist nur sektorspezifische Aufgaben wahrnehmen und vor allem die Kooperation der Staaten organisieren, ging es bei der Europäischen Gemeinschaft von Anfang an um die Integration sowohl der Mitgliedstaaten als auch der Bürgerinnen und Bürger. Zudem wurde sie aufgabenoffen konzipiert; sie kann also verschiedene Aufgaben wahrnehmen, wenn die Mitgliedstaaten ihr diese zuweisen.

Nach der Gründung der Europäischen Wirtschaftsgemeinschaft mit den Römischen Verträgen 1958 wurde das erste Gemeinschaftsprojekt, die Errichtung einer Zollunion, zügig in Angriff genommen. Der weitere Ausbau und die Kompetenzen der Gemeinschaft waren jedoch zwischen Deutschland und Frankreich umstritten. Unter Präsident de Gaulle favorisierte Frankreich ein »Europa der Staaten, aber kein Europa über den Staaten $\iota^{1}{ }^{1}$ Die Mitgliedstaaten sollten über die zentralen Kompetenzen verfügen, während den Gemeinschaftsinstitutionen eine eher dienende Rolle zugewiesen werden sollte. Nach dem Scheitern der Fouchet-Pläne, die diese Vorstellungen umsetzen sollten, begannen in Frankreich und Deutschland Überlegungen, wie sie die Integration vorantreiben könnten. ${ }^{2}$ De Gaulle war weiterhin an einer starken Position Frankreichs in einer von den Mitgliedstaaten dominierten Gemeinschaft gelegen, Bundeskanzler Adenauer wollte den Ausgleich mit Frankreich vorantreiben und Deutschland auf Augenhöhe mit den übrigen Ländern des Westens bringen. Da seine Amtszeit ablief und er seinem potentiellem Nachfolger Ludwig Ehrhard, der als "Atlantiker" eine enge Freundschaft mit den Vereinigten Staaten bevorzugte, misstraute, wollte er einen raschen Abschluss eines Vertrags. ${ }^{3}$

Manche hehren Ziele drohen schon im Keim zu ersticken. Zunächst konnten sich beide Seiten nicht einigen, wer den ersten Schritt gehen sollte. Ein Treffen der Staatsmänner wurde angedacht, aber das Land und die Form der Begegnung waren umstritten. Wer sollte wen besuchen? Schließlich einigten sich beide Seiten darauf, dass Adenauer als Privatmann de Gaulle auf seinem Landsitz bei Colombey-les-Deux-Églises besuchen sollte. Das Treffen am 14. September 1958 wurde ein großer Erfolg und Adenauer blieb der einzige Staatschef, der die Ehre hatte, auf den Landsitz eingeladen zu werden. Der Gegenbesuch in Bad Kreuznach folgte im Jahr darauf. Ein weiterer Besuch de Gaulles 1962 wurde ein Triumphzug. Der Besuch

1 Gerhard Brunn, Die Europäische Einigung von 1945 bis heute (Bundeszentrale für politische Bildung, 2005), 138.

2 Heinrich Siegler, Europäische politische Einigung 1949-1968 (Deutsche Gesellschaft für Auswärtige Politik, 1968), 114-117.

3 Brunn, Die Europäische Einigung, (Fn. 1) $142 \mathrm{f}$. 
beschränkte sich nicht auf die politische Ebene, sondern zog bewusst die Bevölkerung mit ein. Die deutsche Bevölkerung jubelte in Bonn dem französischen Präsidenten zu, und dieser steigerte seine Beliebtheit, indem er deutsch redete und von den Deutschen als einem "großen Volk « sprach. ${ }^{4}$ Diese Worte waren Labsal auf die wunde deutsche Seele. ${ }^{5}$

Im Nachgang dieser Treffen wurde am 22. Januar 1963 im Pariser Elysée-Palast der »Vertrag zwischen der Bundesrepublik Deutschland und der Französischen Republik über die deutsch-französische Zusammenarbeit" unterzeichnet. 18 Jahre nach Kriegsende bekräftigten beide Seiten erstmals ihre Freundschaft und betonten die besonderen Beziehungen zwischen den Ländern. Der Vertrag sieht regelmäßige Konsultationen auf allen Ebenen vor. Der Clou der Vereinbarung besteht darin, dass der Vertrag politische und administrative Aktivitäten anregt und erwartet, sodass sich die Akteure fortan zu permanentem Handeln gezwungen sahen. Keine Seite wollte den erreichten Integrationsstand gefährden und Stillstand wurde als Rückschritt interpretiert, sodass die Integration ständig vorangetrieben wurde. ${ }^{6}$

Der Geist des Elysée-Vertrags besteht in einem Vierklang von Versöhnung, Zusammenarbeit, Solidarität und Freundschaft. ${ }^{7}$ Alle vier Facetten sollten fortan das deutsch-französische Verhältnis prägen. Interessanterweise sollten sie nicht nur die offizielle Kooperation prägen, sondern grundsätzlich das Verhältnis zwischen beiden Ländern auf politischer, aber auch gesellschaftlicher Ebene bestimmen. Diese Zweigleisigkeit spiegelt sich in den Formen der Kooperation wider. So bildeten sich mit der Zeit immer neue gemeinsame Organisationen heraus, darunter der Deutsch-Französische Kulturrat, der Deutsch-Französische Finanz- und Wirtschaftsrat und der Deutsch-Französische Umweltrat. Auf militärischer Ebene wurde der Deutsch-Französische Verteidigungs- und Sicherheitsrat ins Leben geru-

4 Charles de Gaulle, »Rede an die deutsche Jugend «, in Deutsch-Französisches Institut, Über die Freundschaft hinaus... Deutsch-Französische Beziehungen obne Illusionen (Verlag Bonn Aktuell, 1988), 64-66, hier 64.

5 Henri Ménudier, »Der Elyséevertrag von 1963 und die deutsch-französischen Beziehungen «, in Henri Ménudier, Das Deutschlandbild der Franzosen in den 70er Jahren: Gesammelte Aufsätze 1973-1980 (Europa Union Verlag, 1981) 48-57.

6 Hans-Peter Schwarz, Eine entente elémentaire: Das deutsch-französische Verhältnis im 25. Jahr des Elysée-Vertrages (Deutsche Gesellschaft für Auswärtige Politik, 1990).

7 Damit nimmt der Vertrag Gedanken der Schuman-Erklärung vom 9. Mai 1950 auf. Dort heißt es, »Europa lässt sich nicht mit einem Schlag herstellen (...) Es wird durch konkrete Tatsachen entstehen, die zunächst eine Solidarität der Tat schaffen«. Schuman-Plan, abgedruckt in Brunn, Die Europäische Einigung, (Fn. 1) 336-340. 
fen und die deutsch-französische Brigade und das Eurokorps gegründet. ${ }^{8}$ Zentral für die Integration der Bürgerinnen und Bürger wurde das ebenfalls 1963 gegründete Deutsch-Französische Jugendwerk. Knapp 9 Millionen Jugendliche nahmen seitdem an Austauschprogrammen teil. Auf die erste Städtepartnerschaft von 1950 zwischen Ludwigsburg und Montbéliard folgten 2200 weitere Städtepartnerschaft, 4300 Schulpartnerschaften und 22 Regionalpartnerschaften, wie beispielsweise zwischen Thüringen und der Picardie. Mittlerweile gibt es knapp 180 universitäre Doppelabschlüsse, die in beiden Ländern anerkannt werden. Mehr als 1000 Bücher aus Frankreich erscheinen jährlich in Deutschland und 55 französische Filme werden jedes Jahr in den Kinos gezeigt. Außerdem gibt es eine Vielzahl von Ko-Produktionen und mit dem Sender ARTE einen gemeinsamen Vorzeigefernsehkanal. ${ }^{9}$

\section{Das 40-jäbrige Jubiläum}

In den folgenden Jahrzehnten erlangte die Zusammenarbeit zwischen Deutschland und Frankreich eine gewisse Routine. Das gegenseitige Vertrauen wuchs und mit ihm die Früchte der Kooperation. In der Europäischen Gemeinschaft, später Union, wurde der deutsch-französische Motor sprichwörtlich für den Fortgang der Integration. Weitere Schritte zur Vertiefung der EU wurden nur dann möglich, wenn sich Deutschland und Frankreich auf eine gemeinsame Position geeinigt hatten. Konträre Positionen blockierten jeden Integrationsfortschritt, auch wenn die übrigen Mitgliedstaaten sich längst geeinigt hatten. Nach Jahren des stetigen, aber auch ruhigen Ausbaus der deutsch-französischen Zusammenarbeit kam dann anlässlich der Feiern zum 40-jährigen Jubiläum erneut Bewegung in das bilaterale Verhältnis.

Die europäische Politik im ersten Halbjahr 2003 richtete sich vor allem auf drei Bereiche: neue Projekte der deutsch-französischen Zusammenarbeit, die Arbeit im Verfassungskonvent sowie den Irak-Krieg. ${ }^{10}$ Dabei ging Europa durch die tiefsten Wechselbäder seiner neueren Geschichte.

8 Die Institutionen sind abrufbar unter der Startseite www.france-allemagne.fr/Insti tutionen,1131.html (abgerufen am 4. Juni 2018).

9 Zahlen nach Süddeutsche Zeitung 16 (München, 20.-21. Januar 2018); sowie Ronja Kempin, »Neuer Schwung für alte Freunde« (2018) 4-5 Das Parlament www.das-p arlament.de/2018/4_5/im_blickpunkt/-/538186 (abgerufen am 4. Juni 2018), 9.

10 Peter Becker und Olaf Lèße, Die Zukunft Europas: Der Konvent zur Zukunft der Europäischen Union (Springer VS, 2005), 158-172. 


\subsection{Feierlichkeiten und neue Projekte}

Höhepunkt der deutsch-französischen Annäherung waren zweifellos die Feierlichkeiten am 22. Januar 2003, bei denen die Regierungen und Parlamente beider Länder im Prunkschloss von Versailles ihre Freundschaft zelebrierten. Die Feierlichkeiten zum 40. Jahrestag des Elysée-Vertrages waren Anlass für den Deutschen Bundestag, geschlossen nach Versailles zu fahren und dort angemessen an die Überwindung der Erbfeindschaft durch die deutsch-französische Freundschaft zu erinnern. Dafür erwies sich Versailles mit seinen historischen Vorbelastungen als geeigneter, geschichtsmächtiger Ort. Hier, wo Otto von Bismarck nach dem Sieg gegen Frankreich den preußischen König zum Deutschen Kaiser ausrief und Deutschland nach der Niederlage im 1. Weltkrieg die Kapitulationsurkunde unterzeichnen musste, wurde ein negativ besetzter Erinnerungsort zu einem positiven umgedeutet. Ein Lehrstück in Sachen Geschichtsmetaphysik.

Die Feierlichkeiten - auch im Rahmen des ersten Treffens des Deutschfranzösischen Ministerrates - wurden begleitet durch neue Initiativen, die das bilaterale Verhältnis weiter vertiefen sollte. So wurden in den folgenden Jahren fünf Eurodistrikte gegründet, die die Kooperation in den Grenzregionen zwischen Städten und Gemeinden in Elsass/Lothringen und Baden-Württemberg/Rheinland-Pfalz vertiefen sollen. Es wurden Vorschläge lanciert, gemeinsame Kandidaten für internationale Sportverbände aufzustellen sowie langfristig eine doppelte Staatsbürgerschaft für die jeweils im Nachbarland lebenden Deutschen und Franzosen zu vergeben. Die früher so unterschiedlichen Nationen sollen ihre Differenzen einebnen und zu einer, wie es die gemeinsame Erklärung zum 40. Jahrestag des Elysée-Vertrages etwas pathetisch formuliert, "Schicksalsgemeinschaft« in Europa werden. Diese Vorschläge wurden danach nicht weiter verfolgt. Umgesetzt wurde dagegen der Vorschlag zur Erarbeitung eines gemeinsamen deutsch-französischen Geschichtsbuchs.

Was die Europäer allgemein und Deutsche und Franzosen im Besonderen voneinander trennte, waren weniger die unterschiedlichen politischen, administrativen und rechtlichen Systeme, deren Angleichung im Zuge der Integration weiter voranschritt, sondern vor allem die unterschiedlichen geschichtlichen Erfahrungen, die die nationalen Kollektive gemacht haben. Ereignisse, insbesondere der jüngeren Vergangenheit, wurden aus dem jeweiligen nationalen Blickwinkel interpretiert und über Erzählungen, Narrationen und Mythen perpetuiert. In dem Wissen um die Bedeutung, die das kollektive Gedächtnis für die Identität einer Gruppe hat, wurde, wie zuvor erwähnt, im Juni 2003 vorgeschlagen, ein deutsch-fran- 
zösisches Geschichtsbuch zu erarbeiten. ${ }^{11}$ Dieser Vorschlag wurde bis zum Jahr 2011 schrittweise umgesetzt. Es war der erfolgreiche Versuch, die national fixierte Geschichtsschreibung zu durchbrechen, und das nicht nur für einen kleinen Kreis historisch interessierter Intellektueller, sondern auf breitenwirksamer Basis. Das Geschichtsbuch wurde für die Allgemeinheit und die Verwendung in den Schulen beider Länder erarbeitet.

Bis dahin gab es keine für die Allgemeinheit geschriebene, länderübergreifend anerkannte Darstellung einzelner historischer Ereignisse oder der Entwicklung von bestimmten Regionen. Besonders schmerzlich wurde dieser Mangel gerade bei der Bewertung des Zweiten Weltkriegs und seiner Folgen. Gerade bei diesen einschneidenden Ereignissen, Verfolgung, Holocaust und Vertreibung, behielt sich jedes Land seine eigene nationale Interpretation vor und reagierte empfindlich auf Einflussnahme von außen. Das deutsch-französische Geschichtsbuch wurde zum Vorbild für ein ähnliches Projekt zwischen Deutschland und Polen. ${ }^{12}$ Vielleicht wird es einmal eine kontinentübergreifende, allgemein anerkannte Geschichte Europas geben, die an der Ausbildung gemeinsamer Geschichtsbilder mitwirkt und die Unionsbürgerinnen und Unionsbürger dazu bringt, ihre national geprägte Identität wenigstens ein Stück weit aufzugeben, um sie in einen größeren europäischen Kontext einzubetten. Noch ist Europa im Gedenken getrennt.

\subsection{Der deutsch-französische Motor im Verfassungskonvent}

Nachdem bereits im Vorjahr, 2002, der seit langem schwelende Streit um die Landwirtschaftspolitik der Europäischen Union, der bis dahin als beinahe unlösbar galt, beigelegt wurde, ist der deutsch-französische Motor wieder überraschend angesprungen. ${ }^{13}$ Nicht zufällig waren es diese beiden Länder, die ihre Außenminister im Spätsommer 2002 zuerst in den Brüsse-

11 Es sind vier Bände von Histoire/Geschichte im Ernst Klett Verlag (2006-2011) erschienen, die die europäische Geschichte von der Antike bis zur Gegenwart behandeln.

12 Die Webseite der Gemeinsamen DeutschPolnischen Schulbuchkommission ist abrufbar unter http://deutsch-polnische.schulbuchkommission.de/home.html (abgerufen am 4. Juni 2018).

13 Jochim Schild, "Pariser Pragmatismus: Französische Vertragsreformpolitik von Amsterdam bis Lissabon", in Olaf Leiße (Hrsg.), Die Europäische Union nach dem Vertrag von Lissabon (Springer VS, 2010); Gisela Müller-Brandeck-Bocquet, Frankreichs Europapolitik (Springer VS, 2004). 
ler Verfassungskonvent entsandten, auf dem sich die Europäer eine neue politische Form geben wollten. ${ }^{14}$ Als Frucht ihrer Zusammenarbeit haben Staatspräsident Chirac und Bundeskanzler Schröder am 15. Januar 2003 nach einem informellen Treffen einen gemeinsamen Standpunkt zur konstitutionellen Entwicklung der Europäischen Union vorgelegt, der von seinen Urhebern zu Recht als eine "ganz wichtige Schwungradinitiative « bezeichnet worden ist. Die Arbeiten des Konvents zur Zukunft der Europäischen Union, die bis dahin noch zu keinen konkreten Ergebnissen gelangt waren, sollte durch gemeinsame Vorstöße in den Bereichen Außenund Sicherheitspolitik sowie Innen- und Justizpolitik vorangetrieben werden. ${ }^{15}$ Deutschland und Frankreich machten zu diesem Zweck einen Vorschlag zur Schaffung eines EU-Außenministers und einer Doppelspitze aus Rats- und Kommissionspräsidentschaft, die jeweils für fünf Jahre gewählt werden und gemeinsam die Union führen sollen. Aus Brüssel signalisierte der Konventspräsident, der ehemalige französische Staatschef Valérie Giscard d'Estaing, seine Zustimmung zu diesem so genannten »Doppelkopf « und nahm ihn in die Agenda des Konvents auf. Allerdings ist dann später nur der »Außenminister« als Hoher Repräsentant in den endgültigen Entwurf übernommen worden. ${ }^{16}$

Frankreich und Deutschland setzten sich im Rahmen der Innen- und Justizpolitik für die Einrichtung einer europäischen Staatsanwaltschaft und einer europäischen Grenzpolizei ein. Die Grenzschutzagentur Frontex nahm zum Mai 2005 ihre Arbeit auf und gewann durch die Migration aus den südlichen Anrainerstaaten zusätzliches Gewicht. ${ }^{17}$ Die Einrichtung einer Europäischen Staatsanwaltschaft mit Sitz in Luxemburg wurde dagegen erst 2017 von 20 Mitgliedstaaten im Rahmen der Verstärkten Zusam-

14 Die Dokumente des Konvents sind abrufbar unter http://european-convention.eu ropa.eu/DE/bienvenue/bienvenue390c.html?lang=DE (abgerufen am 4. Juni 2018); Becker und Leiße, Die Zukunft Europas (Fn. 10); Colette Mazzucelli, Ulrike Guérot und Almut Metz, »Cooperative Hegemon, Missing Engine of Improbable Core? Explaining the French-German Influence in European Treaty Reform«, in Derek Beach (Hrsg.), Leadership in the Big Bangs of European Integration (Palgrave Macmillan, 2006).

15 Joachim Schild, Französische Positionen in der ersten Phase des EU-Konvents: Raum für deutsch-französische Gemeinsamkeiten? (Stiftung Wissenschaft und Politik, 2002).

16 Becker und Leiße, Die Zukunft Europas (Fn. 10), 162.

17 Die Webseite der Europäischen Agentur für die Grenz- und Küstenwache (Frontex) ist abrufbar unter https://europa.eu/european-union/about-eu/agencies/fronte x_de (abgerufen am 4. Juni 2018). 
menarbeit beschlossen. ${ }^{18}$ Ihre Aufgabe wird die Koordinierung polizeilicher Ermittlungen über nationale Grenzen hinweg, die rasche Einfrierung und Beschlagnahmung von Vermögenswerten im Betrugsfall sowie die Festnahme von Verdächtigen sein. Die Umsetzung dieses deutsch-französischen Vorschlags hat somit 15 Jahre gedauert.

Ferner favorisierten beide Länder im Konvent die Ausweitung der Beschlussfassung mit qualifizierter Mehrheit, die sich auch Vertreter zahlreicher anderer Staaten wünschten. Darüber hinaus kam die Einführung einer Solidaritätsklausel im Falle eines militärischen Konflikts oder terroristischen Anschlag in die Diskussion. Briten und Dänen blieben jedoch die schärfsten Kritiker einer Gemeinsamen Außen- und Sicherheitspolitik (GASP). Beide Länder sprachen sich vehement für einen Verbleib dieses Bereichs bei den Nationalstaaten aus. Eine Position, der sich auch konservativ orientierte Vertreter anderer Staaten anschlossen. Einig waren sich alle Redner, dass die Einheit der Europäischen Union nach außen gestärkt werden müsse, aber über den Weg dorthin waren und blieben sie weiterhin höchst unterschiedlicher Auffassung.

Das galt auch für die europäische Symbolpolitik, die im Konvent lebhaft diskutiert wurde. Ebenso wie Geschichtsauslegung und -schreibung in Europa noch weitgehend national geprägt sind, definieren die Staaten auch selbst ihre nationalen Feiertage. Im Bewusstsein der symbolischen Bedeutung solcher Feiertage wurde vom Verfassungskonvent im Juli 2003 vorgeschlagen, dass Europa einen gemeinsamen Feiertag begehen solle. Der Feiertag soll alljährlich am 9. Mai unter dem Motto »Einig durch Vielfalt« begangen werden und nach Möglichkeit für die Unionsbürgerinnen und Unionsbürger arbeitsfrei sein. Der Vorschlag, der bei den Mitgliedstaaten auf wenig Resonanz stieß, reiht sich ein in die durch die Europäische Union nun forcierte symbolische Politik. Die Umsetzung eines europäischen Feiertags ist bis heute nicht erfolgt, allerdings gibt es nach wie vor die in allen Mitgliedstaaten gleichzeitig durchgeführte Europawoche. Der Verfassungsentwurf legte auch fest, dass die EU neben der bereits bekannten Europa-Fahne auch über eine Hymne, Beethovens Ode an die Freude, verfügen solle. Im Vertrag von Lissabon sind die europäischen Symbole auf Druck Großbritanniens wieder gestrichen worden, doch werden sie im politischen Alltag selbstverständlich verwendet.

18 Europarat, »20 Mitgliedstaaten bestätigen die Errichtung der Europäischen Staatsanwaltschaft « (Pressemitteilung 580/17, 12. Oktober 2017), www.consilium.euro pa.eu/de/press/press-releases/2017/10/12/eppo-20-ms-confirms/pdf (abgerufen am 4. Juni 2018). 
Während der Konvent in die Zielgerade einbog, unterzeichneten am 16. April 2003 zehn Beitrittskandidaten aus Mittel- und Osteuropa sowie dem Mittelmeerraum die Verträge zum Beitritt zur Europäischen Union anlässlich des Gipfels in Athen. Mehr als zwei Stunden dauerte die Unterzeichnung vor laufenden Kameras, die die über 40-jährige Spaltung des Kontinents endgültig überwindet und Europa zum größten Wirtschaftsraum der Welt werden lässt. In Sichtweite der Akropolis, auf der die Säulen des Parthenontempels die anwesenden Staats- und Regierungschefs an die Geburt der Demokratie in Europa erinnerte, wurden die Staaten Europas darauf hingewiesen, dass sie zur Verwirklichung einer europäischen, supranationalen Demokratie noch enger zusammenarbeiten und die Gemeinschaftsinstitutionen stärken müssen. Mit Beendigung des Brüsseler Verfassungskonvents besaß Europa nach harter 16-monatiger Arbeit eine Verfassung, die zwar in den späteren Referenden in Frankreich und den Niederlanden 2005 wieder gekippt worden war, aber 2009 weitgehend unverändert als Vertrag von Lissabon zur aktuell gültigen Rechtsgrundlage geworden ist. Frankreich und Deutschland waren die Schrittmacher im Konventsprozess, ihre Einigkeit bewirkte, dass es mit dem Verfassungsvertrag ein greifbares Konventsergebnis gab.

Im Konvent saßen die Abgesandten aller europäischen Mitgliedstaaten und Beitrittskandidaten sowie Mitglieder des Europäischen Parlaments. Insgesamt 105 politische Repräsentanten, Delegierte unterschiedlicher Nationen, nördlicher und südlicher, westlicher und östlicher Staaten, Vertreter aus Altmitgliedern der Union und den Beitrittskandidaten, reichen und armen Staaten, großen und kleinen, alten, seit Jahrhunderten bestehenden und gefestigten Nationalstaaten ebenso wie erst kürzlich durch die revolutionären Umbrüche im Osten des Kontinents entstandene Staaten. Das gesamte Parteienspektrum war vertreten, von Links- bis Rechtsaußen, Pro-Europäer und Europa-Skeptiker, Föderalisten und Intergouvernementalisten, Männer und (sehr wenige) Frauen, Regierungsvertreter und Parlamentarier. Es war nicht zuletzt ein Erfolg der deutsch-französischen $\mathrm{Zu}$ sammenarbeit, die durch die Jubiläumsfeiern zum Elysée-Vertrag an Schwung gewonnen hatten, dass der Konvent erfolgreich gearbeitet hat und den Verfassungsvertrag als Ergebnis seiner Arbeit dem Europäischen Rat der Staats- und Regierungschefs am 19. und 20. Juni auf ihrem Gipfeltreffen in der Nähe von Thessaloniki vorlegen konnte. ${ }^{19}$

19 Zur Agenda, zum Aufbau und der Arbeitsweise des Konvents über die Zukunft der EU vgl. den Beitrag von Teija Tiilikainen in diesem Band. 


\subsection{Das Schicksalsjahr 2003: Europas außenpolitisches Zerwürfnis}

Der demonstrative Schulterschluss zwischen Deutschland und Frankreich anlässlich der Jubiläumsfeiern führte nicht überall zu vorbehaltloser $\mathrm{Zu}$ stimmung. ${ }^{20}$ Die Reaktionen der »übrigen Welt« ließen nicht lange auf sich warten und schlugen dem freundschaftsseligen Duo überraschend deutlich entgegen. Am 22. Januar 2003 platzte mitten in die Feierlichkeiten in Versailles der fast legendär gewordene Ausspruch des amerikanischen Verteidigungsministers Rumsfeld, Deutschland und Frankreich bildeten das »alte Europa«, das die Zeichen der Zeit nicht erkannt hätte. ${ }^{21}$ Sie seien ein »Problem « im Gegensatz zum »neuen Europa«, das Amerikas geostrategische Interessen, insbesondere hinsichtlich des bevorstehenden Kriegs im Irak, teile. Er lobte explizit die Staaten Mittel- und Osteuropas für ihre Zustimmung zur amerikanischen Irak-Politik und sah in diesen Ländern den zukünftigen Schwerpunkt Europas. Interessant ist die bei den Äußerungen Rumsfelds implizierte Wahrnehmung, dass die kritisierten Länder, die sich untereinander lange als so verschieden charakterisierten, aus der transatlantischen Sicht offensichtlich doch als eine gewisse Einheit wahrgenommen werden.

Die gemeinsame Kategorisierung von Deutschland und Frankreich, die anlässlich der ostentativen Beschwörung der Freundschaft kaum verwundern dürfte, und die Abqualifizierung ihrer gemeinsamen Außenpolitik rief nun eine gemeinschaftliche empörte Reaktion der Kritisierten hervor, die sich gegen die Vorwürfe vehement zur Wehr setzten und nun ihrerseits die Kritiker kritisierten. Die Äußerung Rumsfelds kann in einem semantischen Deutungsfeld verstanden werden, in dem »Alt « für veraltet, vergreist und verbraucht, abgelebt, verknöchert und überholt steht und einen Gegensatz zu »Neu« als jung und dynamisch, frisch, blühend und unverbraucht bildet. Genau hier setzte der Entrüstungssturm aus Europa ein. Da nutzte es auch nichts, dass der 70-jährige Rumsfeld seine Äußerung später als Kosewort verstanden wissen wollte. Die Europäer wehrten sich nicht gegen die gewählte Dimension an sich, sondern sie erklärten die durch Amerika vorgegebene Wertigkeit für nichtig und reagierten unisono mit einer semantischen Umdeutung, sahen alt in einer Beziehung mit weise, erfahren, ehrwürdig und stellten zu Recht heraus, dass das alte Europa mit neuen Formen des Zusammenlebens der Völker experimentiert, während

20 Corine Defrance und Ulrich Pfeil, Der Élysée-Vertrag und die deutsch-französischen Beziehungen 1945-1963-2003 (Verlag Oldenbourg, 2005).

21 Becker und Leiße, Die Zukunft Europas (Fn. 10), 162. 
Amerika seinerseits dem alten, veralteten Macht- und Nationalstaatsprinzip anhängt. Darin waren sich die Intellektuellen, wie Jürgen Habermas, Joseph Rovan, Jorge Semprun und Robert Menasse, mit den Politikern einig. ${ }^{22}$ Auch Hans Magnus Enzensberger und Eric Hobsbawm diskutierten öffentlich über die Wurzeln Europas, seine Zukunft und seine Grenzen. Europa, stilisierte Enzensberger dabei seinerseits den alten Kontinent, sei ein »way of life«. Es überraschte, wie übereinstimmend die Reaktionen waren, die die alten Gräben nicht nur zwischen den betroffenen Nationen, sondern auch zwischen Links und Rechts vergessen ließen.

Nachdem die Bush-Administration die Drohkulisse gegenüber dem Irak mit dem später fragwürdig gewordenen Vorwurf der Produktion von Massenvernichtungswaffen verstärkt und eine bewaffnete Auseinandersetzung kaum mehr zu verhindern war, schrieben acht europäische Staaten einen offenen Unterstützungsbrief. Zu den Unterzeichnern gehörten die großen Drei der EU - Großbritannien, Spanien und Italien - ferner Portugal und Dänemark sowie aus der Reihe der Beitrittskandidaten Polen, Tschechien und Ungarn. Allesamt Länder, die um die neue deutsch-französische Achse herum liegen. Die spanische Außenministerin, Ana Palacio, kritisierte die deutsch-französische Kooperation als »Union innerhalb der Union«, die andere Staaten von ihren Initiativen ausschließe. Die Sympathie der übrigen osteuropäischen Staaten galten ebenfalls den USA, während Griechenland, das die Ratspräsidentschaft inne hatte, Luxemburg, Schweden und Österreich eher dem »alten Europa« zuzurechnen waren. Damit hatten sich bedrohliche Gräben im transatlantischen Verhältnis, innerhalb Europas und im Konvent aufgetan, die sich mit dem näher rückenden IrakKrieg noch vertieften. Und während am 6. Februar Giscard d'Estaing scheinbar unberührt von den weltpolitischen Ereignissen die ersten Artikel sseiner Verfassung dem Konvent präsentierte, griff Chirac die Beitrittskandidaten wegen ihres Haltung in der Irak-Krise scharf an. "Sie haben eine großartige Gelegenheit verpasst, den Mund zu halten«, ließ Chirac verlauten. Und auch der Präsident der Europäischen Kommission, Romano Prodi, zeigte sich besorgt wegen der offen pro-amerikanischen Haltung der Beitrittsstaaten, was zeige, »dass sie noch nicht verstanden hätten, dass

22 Vgl. »Das alte Europa anwortet Herrn Rumsfeld«, FAZ (Frankfurt, 24. Januar 2003). Ein Überblick über die Positionen ist abrufbar unter www.vaovao.net/2003 /Januar/0301-3d/030124fazrumsfeld.htm (abgerufen am 4. Juni 2018). Dazu auch der FAZ-Artikel Jürgen Habermas und Jacques Derrida, »Nach dem Krieg: Die Wiedergeburt Europas«, FAZ (Frankfurt, 31. Mai 2003) www.faz.net/aktuell/feuill eton/habermas-und-derrida-nach-dem-krieg-die-wiedergeburt-europas-1103893.ht ml?printPagedArticle=true\#pageIndex_0 (abgerufen am 4. Juni 2018). 
die EU mehr als eine Wirtschaftsgemeinschaft sei«. Die betroffenen Länder wiesen die Schelte pikiert zurück, und Polen sagte eine Teilnahme an einem Gipfeltreffen von EU-Präsidentschaft und Beitrittsländern ab. ${ }^{23}$

In permanenten Gesprächen mit Russlands Staatschef Putin bildete sich allmählich die bekannte Achse Paris - Berlin - Moskau, später ergänzt um Peking, heraus. Die »Achse der Feiglinge«, wie Rumsfeld verächtlich meinte, während er die »Koalition der Willigen" um sich scharte. Im März 2003 beschleunigten sich die Ereignisse noch einmal. Am 10. März wurde die knappe Zustimmung der sonst eher kritischen Malteser in einem Referendum über den Beitritt zur EU anerkennend zur Kenntnis genommen. Malta eröffnete den Reigen der EU-Referenden in den Beitrittsländern, die allesamt positiv für einen Beitritt zur Union ausfielen. Nach der Rede Präsident Bushs an die Nation 17. März, begann am folgenden Tag offiziell der Irak-Krieg. Am gleichen Tag wollte der europäische Konvent in Brüssel über die Entwicklung diskutieren, doch der Vorschlag wurde alsbald vom französischen Vorsitzenden abgeschmettert. Am 20. März, zu Beginn der Kampfhandlungen im Irak, gab der Europäische Rat eine Erklärung heraus, die sich vor allem mit der Zeit nach dem Ende des Krieges und der als wahrscheinlich angesehenen Niederlage Husseins beschäftigte. ${ }^{24}$ Jedoch war die Erklärung sehr unverbindlich gehalten; konkrete Maßnahmen wurden nicht erwähnt. Es war der kleinste gemeinsame Nenner, auf den sich die Kontrahenten einigne konnten. Die Stimmung zwischen Jacques Chirac und Tony Blair war auf dem Tiefpunkt. Doch steckte dahinter nicht nur eine Meinungsverschiedenheit zwischen zwei Politikern, sondern eine Auseinandersetzung zwischen unterschiedlichen Modellen für Europa. Frankreich bevorzugte die gaullistische Variante eines von Frankreich geführten starken Europas als geopolitischem Gegengewicht zu den die USA, während Großbritannien die Vision eines Markteuropas verfolgte, das in enger transatlantischer Partnerschaft zu den USA steht und gemeinsam gegen die neuen Gefahren vorgeht. Das Gipfeltreffen des Europäischen Rates blieb eine Good-will-Aktion ohne greifbare Annähe-

23 Becker und Leiße, Die Zukunft Europas (Fn. 10), 163. Dazu auch Joschka Fischer, I am not convinced: Der Irak-Krieg und die rot-grünen Jahre (Kiepenheuer \& Witsch, 2011), 207-210. Alyson J.K. Bailes, »The European Security Strategy: An Evolutionary History« (2005) SIPRI Policy Paper 10, 5ff www.sipri.org/publications/ 2005/sipri-policy-papers/european-security-strategy-evolutionary-history (abgerufen am 4. Juni 2018).

24 Europäischer Rat, »Schlussfolgerungen des Vorsitzes« (Brüssel, Tagung vom 20.21. März 2003, 8410/03, 5. Mai 2003) www.consilium.europa.eu/media/20850/75 146.pdf (abgerufen am 4. Juni 2018). 
rung. Noch am gleichen Tag regten Frankreich, Deutschland und Belgien die Aufstellung gemeinsamer Streitkräfte als Kern einer europäischen Armee an. Trotz aller internen Differenzen übernahm die Europäische Union am Ende des Monats mit der Friedensmission in Mazedonien erstmals in ihrer Geschichte ein militärisches Kommando. 350 Soldaten sollten die Waffenruhe zwischen Albanern und Mazedoniern sichern helfen.

Nur wenige Tage nach dem Einmarsch der Amerikaner in Bagdad am 9. April 2003 zelebrierten Frankreich, Deutschland und Russland am 11. und 12. April ihre neue Einigkeit beim Gipfel in St. Petersburg. Die drei Staaten appellierten an die internationale Gemeinschaft, die Vereinten Nationen zu stärken und zu den Grundwerten des Zusammenlebens zurückzukehren. Doch waren Briten und Amerikaner noch zu sehr im Irak involviert, als dass sie die Botschaft vernommen hätten. Außerdem ging von dem Gipfel kein sonstiges positives Signal aus. Die Achse Paris - Berlin Moskau erwies sich als ad hoc gezimmertes Bündnis, das außer dem Wunsch nach Mitsprache bei der sich abzeichnenden Nachkriegsordnung in einem Land, in dem sie nichts zu sagen haben, und die Sorge vor weiterer Marginalisierung wenig einte. Am 29. April trafen sich schließlich Vertreter aus Frankreich, Deutschland, Belgien und Luxemburg, um Möglichkeiten einer Bündelung der Verteidigungspolitik zu sondieren. Ergebnis der Gespräche war der gemeinsame Wunsch, Europas Verteidigung zu stärken. Zu diesem Zweck sollte einerseits eine entsprechende Initiative in den Verfassungskonvent eingebracht werden, die eine verstärkte Zusammenarbeit einzelner Staaten ermöglichen sollte, andererseits wurden schon konkrete Schritte dorthin festgelegt. Der Wille zum Aufbau eines Generalstabes ohne Rückgriff auf Mittel und Fähigkeiten der Nato, die Einrichtung eines strategischen Lufttransportkommandos sowie die gemeinsame Abwehr von atomaren, biologischen und chemischen Waffen wiesen in die angestrebte Richtung. Belgiens Regierungschef Verhofstadt sprach dabei sogar von einem gemeinsamen EU-Hauptquartier und dem Aufbau einer EU-Interventionsarmee. Doch der so genannte »Pralinengipfel« erwies sich als erneuter Alleingang. ${ }^{25}$ Trotz des Angebots an andere Länder, sich an den konkret vereinbarten Maßnahmen zu beteiligen, reagierten diese brüskiert. Selbst die sonst so integrationsfreundlichen Niederländer fehlten. Italien wollte zusammen mit Spanien und Großbritannien einen eigenen Gipfel organisieren, während Tony Blair in London davor warnte, die Vormachtstellung der USA in Frage zu stellen. Die Forderung nach einer führenden Weltmacht USA war natürlich ein Affront ge-

25 Becker und Leiße, Die Zukunft Europas (Fn. 10), 165. 
gen die französischen Vorstellungen von einer multipolaren Welt und vertieften noch einmal die bekannten Gräben.

Doch mit dem von Präsident Bush am 1. Mai 2003 auf dem Flugzeugträger Abraham Lincoln proklamierten offiziellen Ende des Irak-Feldzugs kam erneut Bewegung in die europäische Staatenwelt. Die Ereignisse kreuzten sich zunehmend auf supranationaler, multilateraler und transatlantischer Ebene. Am 2. und 3. Mai trafen sich die EU-Außenminister zu einem informellen Treffen in der Ägäis. Sie beauftragten den außenpolitischen Vertreter der EU, Javier Solana, eine neue globale Sicherheitsstrategie für die Gemeinschaft auszuarbeiten. ${ }^{26}$ Darin soll Europa seine Sicherheitsinteressen definieren und Möglichkeiten zum Aufbau demokratischer Strukturen in der Welt ausloten. Dies habe bis Jahresende zu erfolgen. Mitten hinein in den Abschluss des Treffens platzte die Nachricht, dass Polen neben Briten und Amerikanern einen Sektor im besetzten Irak zukünftig mitverwalten soll. Das Angebot der USA, die damit ihren langjährigen Partner in besonderer Weise auszeichneten, machte den »Alt-Europäern" bewusst, dass die Achse Paris-Berlin, deren Entscheidungen, wie beim Agrarkompromiss des Vorjahres, alle anderen EU-Mitgliedstaaten bedingungslos folgen müssen, nicht von allen europäischen Staaten gutgeheißen wurde. ${ }^{27}$ Polen als größter Beitrittsstaat wies das Führungsduo der Union darauf hin, Politik in der Union besser abzustimmen und mit allen Partner auf gleicher Augenhöhe zu besprechen. Im Übrigen rief Außenminister Bartoszewski dazu auf, die Spaltung in Europa zu überwinden und zu neuer Einheit zu finden. Zu diesem Zweck machte Polen nur wenige Tage später das Angebot, deutsche und dänische Truppen der von den drei Staaten gemeinsam unterhaltenen "Multinationalen Korps Nordost « in Stettin zu beteiligen. Doch schlugen sowohl Deutsche als auch Dänen, die noch im Januar den Brief der Acht $^{28}$ zur Unterstützung der USA mit unterzeichnet hatten, die Offerte aus. Polen musste lernen, dass nach seiner Unterzeichnung des Briefs der Acht der Weg zu einer Annäherung lang sein

26 Antonio Missiroli, "From Copenhagen to Brussels: European Defence - Core Documents (2003) Chaillot Papers 67, European Union Institute for Security Studies www.iss.europa.eu/content/copenhagen-brussels-european-defence-coredocuments-volume-iv (abgerufen am 4. Juni 2018); Ronald D. Asmus, »The European Security Strategy: An American View«, in Roland Dannreuther und John Peterson (Hrsg.), Security Strategy and Transatlantiv Relations (Routledge, 2006).

27 Becker und Leiße, Die Zukunft Europas (Fn. 10), 165.

28 "Offener Brief der acht EU-Länder zum Irak im Wortlaut« (tagesschau.de, 29. August 2007) www.tagesschau.de/ausland/meldung353388.html (abgerufen am 4. Juni 2018). 
kann. Und es musste einsehen, dass eine internationale Aufwertung auch Verantwortung mit sich bringen kann, der man sich erst einmal gewachsen zeigen muss. Beim Gipfeltreffen am 9. Mai im Rahmen des Weimarer Dreiecks überwanden Frankreich, Deutschland und Polen ihr Misstrauen und beschlossen, künftig mehr Initiativen untereinander abzustimmen. Chirac, Schröder und Kwasniewski zeigten demonstrative Einmütigkeit und ließen Beobachter bereits von einer neuen Achse Paris - Berlin - Warschau munkeln. Doch das Treffen blieb letztlich folgenlos. ${ }^{29}$

Im Juni führte der Beschluss, Truppen in den bürgerkriegsgeschüttelten Kongo zu entsenden, die Europäische Union erstmals in einen militärischen Einsatz außerhalb Europas. ${ }^{30}$ Die Führung der EU-Mission unter UN-Mandat übernahm Frankreich, das 1.000 der insgesamt 1.400 Soldaten stellte. Auch Deutschland beteiligte sich nach einigem Zögern schließlich mit der Entsendung von 350 Soldaten, die im benachbarten Uganda operieren sollen. Die Art und Weise, in der dieser Einsatz durch die französische Regierung politisch vorbereitet wurde, zeigt deutliche Anzeichen einer Fortsetzung des Irak-Konflikts. Angesichts der Tatsache, dass der Konflikt im Kongo schon viele Jahre schwelte, immer wieder zu eruptiven Ausbrüchen geführt und dabei seit 1997 über drei Millionen Menschen den Tod gebracht hat, liegt der Verdacht nahe, dass Frankreich die Kongo-Mission gerade zu diesem Zeitpunkt offensichtlich für eigene Zwecke instrumentalisierte. Frankreich demonstrierte, wie im Verein mit den Vereinten Nationen ein EU-Einsatz ohne Rückgriff auf Mittel und Fähigkeiten der Nato aussehen kann. Damit füllte Frankreich den Begriff der gemeinsamen europäischen Sicherheits- und Verteidigungspolitik, der bislang weitgehend politische Absichtserklärung blieb, mit Realpolitik.

\subsection{Die turbulenten Folgen der Elysée-Feiern}

Die Feierlichkeiten zum 40-jährigen Bestehen des Elysée-Vertrags haben Frankreich und Deutschland wieder einander angenähert. Nachdem der deutsch-französische Motor zuvor ins Stottern geriet, haben die Feiern

29 Kai-Olaf Lang und Daniela Schwarzer, Das Weimarer Dreieck jetzt stärken und Nutzen. Europapolitische Funktionen der deutsch-polnisch-französischen Zusammenarbeit (Stiftung Wissenschaft und Politik, 2011). Ergebnisse der Treffen sind abrufbar unter www.weimarer-dreieck.eu (abgerufen am 4. Juni 2018).

30 Muriel Asseburg, Die EU als strategischer Akteur in der Sicherheits- und Verteidigungspolitik? Eine systematische Bestandsaufnahme von ESVP-Missionen und-Operationen (Stiftung Wissenschaft und Politik, 2009). 
wieder neuen Schwung in die Beziehungen gebracht. Nicht nur die bilateralen Beziehungen wurden vertieft, sondern auch die Arbeit im Konvent wesentlich vorangetrieben. Damit trat ein in Europa typisches Muster wieder deutlicher zutage. Wenn Deutschland und Frankreich eng kooperieren, neue Zukunftsprojekte entwerfen und ganz allgemein ihre bilateralen Beziehungen in einem guten Zustand sind, dann profitiert davon auch die europäische Integration. Im Verfassungskonvent konnten beide Länder wesentliche Prozesse anstoßen und ihre Vorstellungen einbringen und weitgehend durchsetzen. Die deutsch-französischen Vorschläge wurden von den übrigen Teilnehmerstaaten dankbar aufgegriffen, sorgfältig diskutiert und in weiten Teilen unterstützt. Deutschland und Frankreich erwiesen sich als gemeinsames Führungsduo in Europa.

Ganz anders sah die Situation in Bezug auf die GASP aus. Hier vermochten es die Partner nicht, die übrigen europäischen Staaten hinter sich zu vereinigen. Nur eine Woche nach den pompösen Feierlichkeiten in Versailles und dem neuen Wir-Gefühl in der Abwehr amerikanischer Kritik haben acht europäische Staaten einen Aufruf zur Unterstützung von George W. Bush im Irak-Konflikt veröffentlicht. ${ }^{31}$ Der Aufruf war nicht nur eine Demonstration europäischer Solidarität mit den USA, sondern zugleich ein Affront gegen das auftrumpfende Selbstbewusstsein der deutsch-französischen Achse. Es handelte sich um ein Zweckbündnis bestimmter Nationen, die der weiteren Integration in Europa kritisch gegenüber standen und langfristig eher einen lockeren Staatenbund anstrebten. Ebenfalls unübersehbar, dass zu den Unterzeichnern gerade die um die neue Achse liegenden Staaten gehörten. Der »Pralinengipfel « verschärfte diese Situation noch weiter. Die Äußerungen Rumsfelds konnten eine Spaltung der Europäer gerade im sensiblen Bereich der Außenpolitik hervorrufen. ${ }^{32}$ Sein ausdrückliches Lob der Staaten Mittel- und Osteuropas für ihre Zustimmung zur amerikanischen Irak-Politik und seine Vermutung, dass hier das neue Europa zu finden sei, hat die durch den europäischen Verfassungskonvent geförderte Einigkeit erfolgreich konterkariert.

Als Fazit zu den Ereignissen des Jahres 2003 kann festgehalten werden, dass vor allem Deutschland und Frankreich als Führungsduo im Bereich der europäischen Integration erfolgreich waren. Im außenpolitischen Bereich haben sie es dagegen nicht vermocht, die europäischen Bündnispartner auf ihre Linie einzuschwören. Abschottung durch Sonderinitiativen, wie den »Pralinengipfel«, unnötige Bündnisse, wie mit Russland, und

31 »Offener Brief der acht EU-Länder«(Fn. 28).

32 Becker und Leiße, Die Zukunft Europas (Fn. 10), 165. 
die Durchführung von militärischen Aktionen ohne Einbettung in den gesamteuropäischen Kontext, wie bei der Mission im Kongo, sorgten dafür, dass zwar die deutsch-französische Verbindung gestärkt, die europäischen Partner aber nicht überzeugt wurden. Beide Länder mussten daher zukünftig vorsichtiger agieren, wenn sie als Führungsduo in Europa auch wirklich führen und keinen anhaltenden Widerstand in anderen Ländern hervorrufen wollten. Führungsstärke, das mussten beide Länder lernen, erwächst nur durch die Anerkennung der Führung durch die Geführten.

\section{Das 55-jährige Jubiläum des Elysée-Vertrags}

Zum 50-jährigen Jubiläum 2013 riefen Frankreich und Deutschland ein deutsch-französisches Jahr aus, das den Rahmen für zahlreiche Veranstaltungen auf politischer und zivilgesellschaftlicher Ebene bildete. ${ }^{33}$ Am 22. Januar 2013 fuhr die französische Regierung unter François Hollande nach Berlin und es gab erneut eine gemeinsame Sitzung von Bundestag und Nationalversammlung. Noch im gleichen Monat richtete das Eurokorps in Straßburg einen Festakt aus und am französischen Nationalfeiertag, dem 14. Juli, nahm die Luftwaffe an einem Flug über Paris teil. Erstmals seit dem Ende des Zweiten Weltkriegs war damit deutsches Militär in der französischen Hauptstadt präsent. ${ }^{34}$

Beim 55-jährigen Jubiläum im Januar 2018 hatten beide Partner schon einige Routine. Diesmal nahm zunächst eine Delegation der französischen Nationalversammlung unter François de Rugy an einer Sondersitzung des Deutschen Bundestags teil. Darin bezeichnete er beide Nationen als »Familie«. ${ }^{35}$ Am Nachmittag srevanchierter sich dann die deutsche Seite unter der Leitung des Bundestagspräsidenten Wolfgang Schäuble mit einem Besuch der französischen Nationalversammlung. Schäuble würdigte die

33 Mechtild Gilzmer, Hans-Jürgen Lüsebrink und Christoph Vatter (Hrsg.), 50 Jahre Elysée-Vertrag (1963-2013): Traditionen, Herausforderungen, Perspektiven (transcript Verlag, 2014); Guido Thiemeyer, »Eine symbolische Inszenierung: Die bilaterale Freundschaft im Rahmen des Elysée-Vertrages«, (2012) Dokumente - Zeitschrift für den deutsch-französischen Dialog 58.

34 Christophe Braouet, Deutschland - Frankreich. Partner für Europa. 50 Jahre nach dem Elysée-Vertrag (Verlag Dr. Dieter Winkler, 2012). Ulrich Krotz und Joachim Schild, Shaping Europe: France, Germany and Embedded Bilateralism from the Elysée Treaty to Twenty-First Century Politics (Oxford University Press, 2013).

35 Zum Ablauf der Feierlichkeiten Michaela Wiegel und Majid Sattar »Unsere beiden Länder sind eine Familie«FAZ (Frankfurt, 22. Januar 2018) www.faz.net/-gpg -96by8 (abgerufen am 4. Juni 2018). 
deutsch-französischen Beziehungen als »Geschenk aus der Geschichte« und betonte die Verantwortung beider Länder für die europäische Integration: »Als Deutsche und Franzosen wollen wir vorangehen«. Die Feierlichkeiten wurden nur wenig getrübt durch die demonstrative Ablehnung der Besuche durch die deutsche Alternative für Deutschland und den französischen Front National.

In der gemeinsamen Resolution von Deutschem Bundestag und französischer Assemblée nationale mit dem Titel »Für einen neuen Élysée-Vertrag - Die Rolle der Parlamente in der deutsch-französischen Zusammenarbeit stärken" wird ein »neuer Elysée-Vertrag " gefordert. Zahlreiche neue konkrete Projekte werden angedacht und sollen in den nächsten Jahren umgesetzt werden. Dazu zählen unter anderem: ${ }^{36}$

- Vertiefung der grenzüberschreitenden Zusammenarbeit: gegenseitige Anerkennung von Bildungsabschlüssen aus dem schulischen, berufsbildenden und akademischen Bereich, das Erlernen der jeweiligen Nachbarsprache, den Austausch von Personal in Kindertagesstätten und Schulen, medizinische Leistungen im Nachbarland, besserer Datenaustausch und Zusammenarbeit von Bundespolizei und Police aux Frontières (PAF), bessere grenzüberschreitende Schienenverbindungen und Verkehrswege.

- Mehr Kompetenzen für die Eurodistrikte: Übernahme Trägerschaft von grenzüberschreitenden Einrichtungen wie Kindertagesstätten oder Gesundheitseinrichtungen und Betrieb des grenzüberschreitenden öffentlichen Nahverkehrs.

- Deutsch-französischer Wirtschaftsraum: gemeinsame Regelungen beispielweise im Unternehmens- und Konkursrecht; Angleichung der Bemessungsgrundlage der Körperschaftsteuer.

- Jugend, Bildung, Sprache und Kultur: Gründung von deutsch-französischen Berufsschulzentren, mehr Auszubildendenaustausch, Stärkung der deutsch-französischen Zusammenarbeit im Hochschulwesen mithilfe der Harmonisierung des Hochschullehrerstatuts; mehr bilinguale Schulen, in denen die deutsche und die französische Hochschulzugangsberechtigung erworben werden können; mehr Kooperationen zwischen den Instituts français und den Goethe-Instituten.

36 Gemeinsame Resolution von Assemblée nationale und Deutschem Bundestag zum 55. Jahrestag des Élysée-Vertrags am 22. Januar 2018, »Für einen neuen Élysée-Vertrag - Die Rolle der Parlamente in der deutsch-französischen Zusammenarbeit stärken«, www.bundestag.de/blob/537270/a0aac9a1ba8b4ea2867ecb6e9faa1 f35/traite_elysee_resolution-data.pdf (abgerufen am 4. Juni 2018). 
Darüber hinaus sollen Deutschland und Frankreich gemeinsam europäische Impulse in der GASP, im Klimaschutz, im Energiebinnenmarkt, in der Künstlichen Intelligenz und in der Migration ausarbeiten. Im Vergleich zu den zuvor genannten, recht konkreten Projekten wird in diesem Bereich vorsichtiger argumentiert. Die Erfahrungen aus der Entwicklung 2003 und den folgenden Jahren hat die Partner vorsichtiger werden lassen bezüglich außenpolitischer Themen. Die Resolution spricht eher vage von einem Ausbau bestehender Kapazitäten und der Entwicklung einer gemeinsamen strategischen Kultur. Keineswegs beanspruchen die Partner offensiv eine Führungsrolle beim Ausbau der GASP. Nach dem Austritt Großbritanniens aus der Europäischen Union zählen Deutschland und Frankreich zwar zu den größten, militärisch stärksten und daher sicherheitspolitisch relevantesten Mitgliedstaaten, aber zumindest auf dem Papier wird die mögliche zukünftige Führungsrolle zurückhaltend formuliert. Denn noch ist keineswegs sicher, ob die übrigen Mitgliedstaaten in der EU eine deutsch-französische Hegemonie in dem sensiblen Politikfeld der Sicherheitspolitik akzeptieren.

Dagegen wird in der Resolution für mehr Kooperation geworben und diese auch an konkret umsetzbaren Projekten festgemacht. Die Zukunft wird zeigen, welche Projekte wirklich umsetzbar sind. Auffällig gegenüber der Situation 2003 ist, dass die Zahl der Projekte und die Breite der betroffenen Politikfelder erheblich zugenommen haben. Der Erfolg der Kooperation wird sich an der Umsetzung der vorgeschlagenen Projekte messen lassen müssen. Darüber hinaus fordert die Resolution die Einsetzung einer deutsch-französischen Arbeitsgruppe, der Abgeordnete der beiden Parlamente angehören und die einen Vorschlag für ein »Deutsch-Französisches Parlaments-Abkommen « erarbeiten sollen. Diese Arbeitsgruppe, der je neun Abgeordnete beider Parlamente angehören sollen, soll die gemeinsamen Standpunkte in die Verhandlungen der Regierungen über die Aktualisierung des Elysée-Vertrags einbringen. Dazu zählen 18 Punkte, die in der Resolution bereits ausführlich beschrieben werden und die eine enge Kooperation zwischen beiden Institutionen sicherstellen soll.

Inhaltlich steht die Resolution im Kontext einer Reformoffensive für die Europäische Union von französischer Seite. In seiner Rede an der Pariser Sorbonne vom 26. September 2017 bot der französische Staatspräsident Emmanuel Macron Deutschland - nur zwei Tage nach der Bundestagswahl -eine »neue Partnerschaft« an und schlug zugleich eine Reform der 
EU vor. ${ }^{37}$ Mit Deutschland will Frankreichs Staatspräsident eine noch engere Partnerschaft eingehen. Vorstellbar sei, die Märkte beider Länder bis 2024 vollständig zu integrieren. Macron schlug darüber hinaus ein ganzes Bündel an möglichen Zukunftsprojekten für die Europäische Union vor. Dazu zählen institutionelle Reformen, wie die Verkleinerung der Kommission auf 15 Kommissare, die Wahl der Hälfte der Abgeordneten im Europäischen Parlament über länderübergreifende Listen, die Gründung einer europäischen Asylbehörde, eine Geheimdienstakademie, gemeinsamer Katastrophenschutz und eine europäische Agentur für Innovationen. Im Bereich des Binnenmarktes schlug er ein eigenes EurozonenBudget, einen Eurozonen-Finanzminister, die Einführung der Transaktionssteuer und die Angleichung der Unternehmenssteuern vor. Bezüglich der GASP forderte er ein europäisches Verteidigungsbudget sowie die Aufnahme von Soldaten anderer Mitgliedstaaten in die nationalen Armeen.

Die Resolution von Bundestag und Assemblée nationale bereitet die Fortführung der Kooperation im Rahmen eines neuen »Elysée-II-Abkommens« vor. Strategisch geschickt, enthält die Resolution große Visionen und Zukunftsideen, die gleichzeitig mit konkreten Projekten zumindest in der deutsch-französischen Zusammenarbeit unterfüttert werden und zusätzlich enge und konkrete Vorgaben für die institutionelle Zusammenarbeit vorsieht. Die gelungene Mischung aus Idealismus und Realismus erinnert an den Schuman-Plan von 1950 und den Elysée-Vertrag, in dem es neben dem Willen zur Kooperation auch um die Umsetzung nationaler Interessen ging. Beide Ansätze, der idealistische wie der realistische, prägen die bisherigen "Jahrhundertverträge«.

Der neue »Elysée-II-Vertrag« sollte ursprünglich bis zu den Feierlichkeiten zum 55-jährigen Jubiläum ausgehandelt sein, jedoch durchkreuzte die verzögerte Regierungsbildung in Deutschland diesen ehrgeizigen Plan. Stattdessen wurde das Abkommen als »Vertrag von Aachen« zum 56-jährigen Jubiläum am 22. Januar 2019 im Krönungssaal des Aachener Rathauses von Bundeskanzlerin Merkel und Staatspräsident Macron unterzeichnet. Der Vertrag von Aachen deckt eine Vielzahl von Politikbereichen ab, von der Gemeinsame Außen- und Sicherheitspolitik über die innere Sicherheit und die Bildungspolitik bis zur Zusammenarbeit in den Grenzre-

37 Emmanuel Macrons, »Initiative für Europa« (Sorbonne, Paris, 26. September 2017) https:/de.ambafrance.org/Staatsprasident-Macron-Initiative-fur-Europa (abgerufen am 4. Juni 2018). Zur Wahl Macrons: Jocelyn Evans und Gilles Ivaldi, The 2017 French Presidential Elections: A Political Reformation (Palgrave, 2018). 
gionen. ${ }^{38}$ Ergänzend dazu wurden eine Reihe von konkreten Prioritäten, wie beispielsweise die Schaffung von vier integrierten Deutsch-Französischen Kulturinstituten in ausgewählten Drittstaaten sowie die Verbesserung der Verkehrsverbindungen zwischen beiden Ländern, beschlossen. ${ }^{39}$ Und schließlich wird die Zusammenarbeit auf allen politischen und administrativen Ebenen verstärkt. »Beide Staaten halten vor großen europäischen Treffen regelmäßig Konsultationen auf allen Ebenen ab und bemühen sich so, gemeinsame Standpunkte herzustellen und gemeinsame Äußerungen der Ministerinnen und Minister herbeizuführen.« (Art. 2 Vertrag von Aachen).

Mit diesem Dreiklang aus allgemeinen Absichtserklärungen, konkreten Maßnahmen und dem Willen zur Kooperation weist der Vertrag von Aachen in eine neue Ära der deutsch-französischen Zusammenarbeit. Nachdem die Corona-Krise 2020 die Umsetzung zunächst beeinträchtigt hatte und sogar zu zeitweiligen Grenzschließungen führte, konnte die neue Zusammenarbeit einen ersten Erfolg verbuchen. Zur Überwindung der Wirtschaftskrise haben sich Angela Merkel und Emmanuel Macron auf einen milliardenschweren Wiederaufbaufonds für die Zeit nach der Coronakrise geeinigt. Deutlich wird erneut, dass die europäische Einigung, die in der Corona-Zeit schweren Schaden genommen hat, nur durch den deutsch-französischen Motor wieder an Fahrt gewinnt.

\section{Fazit}

Mehr als 55 Jahre nach Abschluss des Elysée-Vertrags kommt wieder Bewegung in die Beziehungen zwischen Deutschland und Frankreich. Über viele Jahre hat der Vertrag die bilateralen Beziehungen gefördert und geprägt. Die Anfangsjahre standen dabei ganz im Zeichen der Versöhnung und der Zunahme der Kooperation in vielen Politikfeldern. Der ElyséeVertrag förderte nicht nur die Zusammenarbeit auf der politischen und administrativen Ebene, sondern er bezog ganz bewusst auch die Zivilgesellschaft und die Unionsbürgerinnen und Unionsbürger durch zahlreiche Austauschprojekte mit ein. Der Vertrag erfüllte damit auch eine Forde-

38 Vertragstext abrufbar unter https://de.ambafrance.org/Text-des-Vertrags-von-Aach en.

39 Deutsch-Französische Agenda: Prioritäre Vorhaben in Umsetzung des Vertrags von Aachen, https://www.bundesregierung.de/resource/blob/973812/1571028/633 c78d6e85c9cf4d93ccdf94b56119e/2019-01-22-prioritaere-vorhaben-vertrag-aachendata.pdf?download=1 (abgerufen am 22. Juni 2020). 
rung des EG-Vertrags von 1957, die Grundlagen »für einen immer engeren Zusammenschluss der europäischen Völker zu legen« (Präambel). ${ }^{40}$ Die schrittweise, prozessuale Integration führte Frankreich und Deutschland in den zurückliegenden Jahrzehnten auf allen Ebenen enger zusammen, von den großen Gesten, wie dem ikonischen Händedruck von Helmut Kohl und François Mitterand auf den Schlachtfeldern von Verdun und die pragmatische Kooperation von Gerhard Schröder und Jacques Chirac über die Verzahnung der politischen Ebenen bis hin zum Austausch von Schülern, Studierenden und Auszubildenden.

Das Jubiläumsjahr 2003 war der Anstoß für die Erneuerung der Zusammenarbeit. Das deutsch-französische Tandem gab im Anschluss an die Feierlichkeiten zum 40-jährigen Jubiläum des Elysée-Vertrags die notwendigen Impulse im Verfassungskonvent, der die neue rechtliche Grundlage für die Europäische Union aushandelte. ${ }^{41}$ Die Außenminister stießen im Konvent höchstpersönlich neue Vorhaben an, und der französische Konventspräsident nutzt seine zentrale Rolle, um die Arbeit des Konvents mit dem Verfassungsvertrag zu krönen. Die außenpolitische Lage 2003 zeigte dem deutsch-französischen Tandem aber auch zugleich seine Grenzen auf. Die übrigen Europäer waren und sind nicht bereit, diesem Duo im außenpolitischen Bereich bedingungslos zu folgen. Hinzu kamen unsensible Äußerungen und Vorgehensweisen insbesondere Frankreichs.

Das Jubiläumsjahr 2018 stellte die Partner vor neue Herausforderungen, die es notwendig machen, einen neuen »Elysée-II-Vertrag« aushandeln. Der Vertrag von Aachen liefert die vertragliche Grundlage für mehr Kooperation zwischen beiden Ländern. Konkrete bilaterale Projekte stehen im Mittelpunkt der Zusammenarbeit, an deren Umsetzung einmal der neue Vertrag gemessen werden wird. Im Vergleich zum Jahr 2003 ist die Zahl der angedachten Projekte erheblich gestiegen. Nunmehr werden nicht nur vereinzelte Projekte vorgestellt, sondern es wird ein breites Tableau präsentiert. Auch wenn nicht alle Projekte umgesetzt werden, wie die Erfahrung aus der Vergangenheit zeigt, beeindruckt die Vielzahl der Projekte und der einbezogenen Politikfelder.

Im Bereich der europäischen Integration wird das deutsch-französische Tandem in den kommenden Jahren ebenfalls zahlreiche Reformen anstoßen. Wie bereits 2003, werden in der Europäischen Union vor allem die-

40 Vertrag zur Gründung der Europäischen Wirtschaftsgemeinschaft [1957] 11957E/ TXT.

41 Peter-Christian Müller-Graff, »Elysée-Vertrag und Europäische Union - eine konzeptionelle Annäherung «, in Daniela Heid, Rüdiger Stotz und Arsène Verny (Hrsg.), Festschrift für Manfred A. Dauses zum 70. Geburtstag (C.H. Beck, 2014). 
jenigen Reformen umgesetzt, auf die sich Deutschland und Frankreich zuvor verständigt haben. Frankreichs Präsident Macron hat eine lange Liste vorgelegt. ${ }^{42}$ Die Verständigung auf die Errichtung eines gemeinsamen kreditfinanzierten Wiederaufbaufonds zur Ankurbelung der Wirtschaft in der Post-Corona-Zeit ist die Frucht einer jahrzehntelangen Kooperation und wird sicherlich die Umsetzung weiterer Projekte erleichtern.

Im Bereich der GASP sind die Reformvorstellungen beider Partner eher vorsichtig. Die Lehre aus dem unrühmlichen Jahr 2003 zeigt, dass sich die Europäer nicht ohne Widerspruch hinter Deutschland und Frankreich stellen und eher auf Souveränität setzen. Daher ist es wichtig, dass in diesem sensiblen Politikbereich alle Mitgliedstaaten mitgenommen werden. Gerade in der wichtigen Frage nach dem Einsatz militärischer Mittel dürfen sich die Europäer nicht spalten lassen. Das Europa des Jahres 2003 schwankte zwischen zwei extremen Polen; Assoziierung und Dissoziierung prägten das Bild der politischen Landschaft. Wie gezeigt, fanden sich Gruppen issue-spezifisch und ad hoc zusammen, trennten sich alsbald und gingen andere Konstellationen ein. Das Europa des Jahres 2018 agiert hingegen trotz aller Differenzen beispielweise in der Finanzkrise und der Migrationskrise stärker gemeinschaftlich.

Aufgabe des Vertrags von Aachen wird es sein, Deutschland und Frankreich tatsächlich zu einer "Familie« werden zu lassen, in der sich beide Partner gleichberechtigt wiederfinden. Der neue Vertrag wird, wie bislang der alte Elysée-Vertrag, die Möglichkeit eröffnen, den europäischen Herausforderungen gemeinsam zu begegnen. ${ }^{43}$ Der Prozess einer - sozialpsychologisch gesprochen - Ingroup-Bildung wird weiter vorangetrieben und umfasst auch die Bürgerinnen und Bürger beider Staaten. ${ }^{44}$ Regieren jenseits des Nationalstaats braucht langfristig diesen Unterbau eines gemeinsamen Wir-Gefühls, sonst bleibt es ein Regieren im politischen Vakuum.

42 Macron, »Initiative für Europa« (Fn. 37); Emmanuel Macron, „Für eine neue europäische Souveränität“ (Europäisches Parlament, Brüssel, 17. April 2018) https:// de.ambafrance.org/Staatsprasident-Macron-vor-dem-Europaparlament-Fur-eine-ne ue-europaische (abgerufen am 4. Juni 2018).

43 Robert Frank, »Der Elysée-Vertrag, ein deutsch-französischer Erinnerungsort?«, in Defrance und Pfeil (Hrsg.), Der Elysée-Vertrag (Fn. 20).

44 Olaf Leiße und Utta-Kristin Leiße, »Auf dem Weg zum Wir: Ein sozialpsychologischer Beitrag zur Europäischen Integration«, in Michael Salewski und Heiner Timmermann (Hrsg.), Gesichter Europas (LIT-Verlag, 2002); Jürgen Habermas, Die postnationale Konstellation: Politische Essays (Suhrkamp, 1998). 
